David Daniel Peña Miranda Doctor en Economía de la Empresa. Profesor e investigador del Grupo de Investigación Turismo Competitividad y Desarrollo Sostenible. Facultad de Ciencias Empresariales y Económicas, Universidad del Magdalena. Magdalena, Colombia.

[ddpena@unimagdalena.edu.co]

antonio Guevara Plaza

Doctor en Ciencias Económicas y Empresariales. Profesor titular de la Facultad de Turismo y director del Grupo de Investigación en Sistemas de Información Cooperativos,

Universidad de Málaga, España.

[guevara@uma.es]

José Antonio Fraiz Brea

Doctor en Ciencias Económicas y Empresariales. Profesor titular de Organización de Empresas y director del Grupo de Investigación OC2 Marketing y Turismo. Universidad de

Vigo, España [jafraiz@uvigo.es]

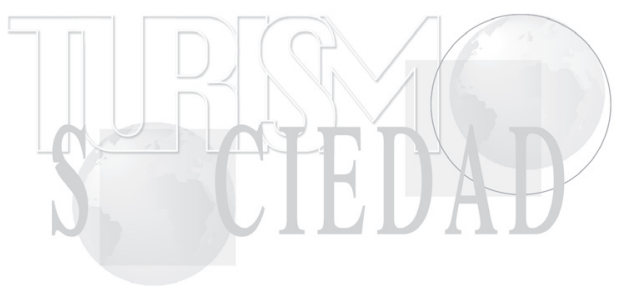

\section{LA INVESTIGACIÓN DE LA RESPONSABILIDAD SOCIAL EMPRESARIAL EN EL SECTOR HOTELERO. ANÁLISIS Y REVISIÓN DE LA LITERATURA CIENTÍFICA ${ }^{1}$}

THE INVESTIGATION OF BUSINESS SOCIAL RESPONSIBILITY IN THE HOSPITALITY SECTOR. ANALYSIS AND REVIEW OF THE SCIENTIFIC LITERATURE

1 Fecha de recepción: 19 de agosto de 2015

Fecha de modificación: 27 de octubre de 2015

Fecha de aceptación: 19 de noviembre de 2015

Para citar el artículo: Peña, D., Guevara, A. y Fraiz, J. (2016). La investigación de la responsabilidad social empresarial en el sector hotelero. Análisis y revisión de la literatura científica. Turismo y Sociedad, XVIII, pp. 137-158. DOI: http://dx.doi.org/10.18601/01207555.n18.08

\section{Resumen}

Durante la última década, los académicos han mostrado un evidente interés en el estudio de la responsabilidad social empresarial (RSE), sin embargo, en la literatura científica son escasos los trabajos sectoriales en el tema, más aún en el sector turístico y, en específico, en el hotelero. Con el objetivo de identificar el estado actual de la investigación de la RSE en el sector hotelero, en el presente trabajo se hace un rastreo sobre los artículos más importantes de revisión bibliográfica en materia de RSE desde el ámbito general del management, seguido de un amplio análisis y revisión de la literatura científica de RSE producida en la industria hotelera durante los últimos diez años. Se concluye que sigue siendo escasa la investigación de la RSE en el sector hotelero, y que sus trabajos han estado dirigidos al análisis de prácticas de RSE, accountability, marketing y la relación con los resultados financieros.

Palabras clave: Responsabilidad social empresarial, turismo, sector hotelero, prácticas, accountability, marketing, resultados financieros.

\begin{abstract}
During the last decade, academics have shown a clear interest in the study of Corporate Social Responsibility (CSR), however, in the scientific literature the sectorial studies and researches on the subject are scarce and even more, in the tourism sector and specifically, in the hotel sector. In order to identify the current state of research on CSR in the hotel sector, in this paper a trace on the most important articles on literature review on CSR from the general scope of Management was done and it was carry out a comprehensive analysis and review of the scientific literature on CSR in the hotel industry produced for the past 10 years. It is concluded that research on CSR in the hotel sector is still scarce and its studies has been directed to the analysis of
\end{abstract}


CSR practices, accountability, marketing and the relationship with financial performance.

Keywords: Corporate social responsibility, tourism, hotel sector, practices, accountability, marketing, financial performance.

\section{Introducción}

En la sociedad actual, el poder y la importancia económica del sector empresarial son evidentes. En un par de estudios interesantes, Glattfelder \& Battiston (2009) y Vitali, Glattfelder \& Battiston (2011), pertenecientes a un grupo prestigioso de teóricos de sistemas complejos de la Escuela Politécnica Federal (ЕTH) de Zurich (Suiza), han estudiado las redes de control y el poder de las empresas en el ámbito mundial, y concluyen que 147 ETN (empresas transnacionales) controlan alrededor del $40 \%$ de la riqueza mundial.

En un trabajo más reciente elaborado por Transnational Institute (2014), se concluye que hoy en día, 37 de las 100 mayores economías del mundo son ETN. De igual manera, según el Informe sobre las Inversiones en el Mundo de 2013, elaborado cada año por la Conferencia de las Naciones Unidas para el Comercio y el Desarrollo (UNCTAD, por sus siglas en inglés), las filiales extranjeras de las ETN generaron ventas por un valor de 26 billones de dólares americanos en 2012, que representan un $36 \%$ del PIB mundial. En términos de crecimiento, esto significa un aumento del 7,4\% con respecto a 2011, mayor al 2,3\% que registró el PIB mundial para el mismo periodo. Así mismo, en términos de empleo, generaron 72 millones de puestos de trabajo, lo que equivale a un incremento del $5,7 \%$ en relación con 2011 (UNCTAD, 2013).

El panorama para el sector turístico no se aleja de esta realidad al demostrar que es uno de los sectores económicos más importantes y con mayores tasas de crecimiento. Según el
Consejo Mundial de Viajes y Turismo (WTTC, por sus siglas en inglés), el turismo representa en el mundo el 9,5\% del producto interno bruto (PIB), el 4,4\% de las inversiones, el 5,4\% de las exportaciones y está empleando al $9 \%$ del total de trabajadores; se estima que en 2024 alcance el 10,3\% del PIB mundial y genere cerca de 74,5 millones de empleo (WTTC, 2014).

Estos datos hacen pensar que las empresas en general, y en particular las que forman parte del sector turístico, tienen todo el potencial para influir de mejor manera en el desarrollo de la sociedad, contribuir así a su bienestar global más eficazmente y, en últimas, a la reconstrucción de un mundo más justo y equitativo para todos.

Infortunadamente, en muchos casos la conducta empresarial no ha sido la esperada por la sociedad, y si este análisis se lleva al plano general de la realidad económica de los últimos cinco (5) años, es evidente que las actuaciones empresariales han sido la principal causa de la que ha sido considerada una de las peores crisis económicas financieras de la historia, la crisis de 2008, de la que muchos países se vienen recuperando lentamente, pero que mantiene fracturada aún a muchas otras economías, tanto de países desarrollados como de países en vía de desarrollo.

Se requiere, entonces, de normas mínimas de conducta empresarial con las que, dentro del libre juego del mercado, las empresas minimicen los impactos sociales y medioambientales negativos que sus actividades conllevan a lo largo y ancho del planeta (De la Cuesta, 2004, citado en Peña y Serra, 2012), haciendo necesario también el planteamiento de un modelo de gestión que convierta a todos los stakeholders o grupos de interés de las organizaciones en la esencia de la estrategia empresarial. La responsabilidad social empresarial (RSE) se convierte en el vehículo para alcanzar este objetivo (Peña y Serra, 2012). 
De manera particular, los establecimientos hoteleros son empresas genuinamente turísticas, ya que ofrecen productos y servicios para el uso explícitamente turístico de los consumidores y están situados geográficamente en el destino turístico (Ayuso, 2003, citado en Peña y Serra, 2012), por lo que sus impactos positivos y negativos son más evidentes en las comunidades de acogida. Por ello, estudiar la RSE en el sector hotelero es de gran utilidad e importancia, y se convierte en el principal objetivo de la presente investigación.

En este sentido, a pesar del evidente interés que el estudio de la RSE ha generado en el mundo académico durante la última década, en la literatura científica son escasos los trabajos y las investigaciones sectoriales en el tema, más aún en empresas del sector turístico, y mucho más en el hotelero.

Partiendo de esta realidad, y con el objetivo de identificar el estado actual de la investigación de la RSE en el sector hotelero, en el presente trabajo se hace un rastreo sobre los artículos más importantes de revisión bibliográfica en materia de RSE desde el ámbito general del management, seguido de un amplio análisis y revisión de la literatura científica de RSE producida en la industria hotelera durante los últimos diez años, de tal forma que se puedan conocer, a ciencia cierta, las líneas específicas de investigaciones sobre las que han versado los diferentes trabajos en la materia.

\section{Metodología}

Una de las definiciones clásicas más utilizadas sobre 'artículo de revisión' ha sido la realizada por Gardfield (1987), en la que expone que es un estudio bibliográfico que recopila, analiza, sintetiza y discute la información publicada sobre un tema, y que puede incluir un examen crítico del estado de los conocimientos reportados en la literatura.
En este sentido, y teniendo en cuenta la clasificación sobre artículos de revisión que Squires (1989) propone, el presente trabajo se encuentra enmarcado dentro de la tipología de revisión descriptiva, ya que proporciona una actualización bibliográfica sobre conceptos útiles en un área en constante evolución, como lo es la RSE, desde el ámbito general del management, pero también desde el ámbito específico del sector turístico y hotelero.

En concreto, la investigación, propiamente dicha, se basó en una revisión de literatura, la cual puede ser definida como el resumen y análisis crítico de artículos, libros y otros documentos que describen el estado de conocimiento sobre el tema por investigar (Creswell, 2002). Autores como Glass, Ramesh \& Vessey (2004) han clasificado la revisión de la literatura como un enfoque de investigación; así mismo, han catalogado el análisis y la revisión de la literatura como un método de investigación.

Por lo tanto, el método de investigación concreto empleado ha sido el análisis y la revisión de la literatura científica, aplicando las tres etapas propuestas por Kitchenham (2004), es decir, (i) planificación de la revisión, (ii) desarrollo de la revisión y (iii) publicación de los resultados de la revisión.

En la etapa de la planificación se identificaron las necesidades de la revisión, se definieron los protocolos de búsqueda y se establecieron los protocolos de revisión. Por su parte, en la etapa del desarrollo se llevó a cabo la revisión propiamente dicha mediante la búsqueda de estudios primarios y la respectiva selección, extracción, gestión y síntesis de la información de interés plasmada en los estudios. Finalmente, la tercera etapa correspondió a la utilización de los resultados y su debida comunicación por medio de la elaboración del presente artículo. 
En la práctica, las publicaciones realizadas en revistas científicas, tesis (principalmente doctorales), centros académicos y consultorías especializadas fueron consultadas.

En relación con las revistas científicas, se consultaron aquellas que estuvieran indexadas/inscritas en bases de datos científicas importantes y listados/índices de reconocimiento científico, entre los que se encuentran, principalmente, Science Citation Index, Social Sciences Citation Index (ambos del ISI - Institute for Scientific Information, que elabora anualmente el Journal Citation Report - JCR); Scimago Journal Ranking (SJR); Scopus; Science Direct; SciELO (Scientific Electronic Library Online); DOAJ (Directory of Open Acces Journals); Google Scholar; Redalyc (Red de Revistas Científicas de América Latina y el Caribe, España y Portugal); Latindex (Sistema Regional de Información en Línea para Revistas Científicas de América Latina, El Caribe, España y Portugal); Dialnet (Portal de difusión de producción científica de España, Portugal y Latinoamérica); DICE (Difusión y Calidad Editorial de las Revistas Españolas de Humanidades y Ciencias Sociales y Jurídicas); Publindex (Sistema Nacional de Indexación y Homologación de Revistas Especializadas de Ciencias, Tecnología e Innovación de Colombia).

También se tuvieron en cuenta revistas no indexadas, pero con cierto reconocimiento científico en el área de estudio, que evidencian objetividad y rigor en el proceso de selección de los artículos, con comités editoriales/científicos destacados y publicaciones periódicas y visibles en otras bases de datos secundarias nacionales o internacionales.

Con base en esto, se procedió a la búsqueda, clasificando las revistas según los siguientes seis (6) criterios: (i) revistas académicas genéricas de management internacionales; (ii) revistas académicas genéricas de management nacionales (España y Colombia); (iii) revistas académicas propias del campo de la RSE; (iv) revistas académicas especializadas en turismo y hotelería internacionales; (v) revistas académicas especializadas en turismo y hotelería nacionales (España y Colombia); (vi) revistas académicas de otras disciplinas que hayan estudiado la RSE.

En relación con las publicaciones de tesis, centros académicos y consultorías especializadas, fueron consultados aquellos documentos cuya temática fuera la RSE, aplicados al management en general y al sector hotelero en particular.

Finalmente, el horizonte de tiempo establecido para el análisis y la revisión de la literatura de la RSE en el sector hotelero se estableció en diez años, es decir, de 2004 a 2014.

Una vez expuesto lo anterior, se procederá a mostrar en los próximos epígrafes los resultados concretos de la revisión.

\section{Investigación académica de la RSE desde el ámbito general del management}

El punto de partida para el análisis de la RSE en la literatura científica desde la época moderna se dio a partir de la publicación del libro Social Responsibilities of the Businessmen, de Howard Bowen (1953), quién propone la RSE como un medio para conseguir los resultados empresariales en función de los objetivos y valores de la sociedad, más allá de las limitadas responsabilidades que se atribuían los directivos de la época (Caroll, 1979). A partir de ahí, el estudio de la RSE comenzó a despertar un interés académico importante que se materializó con el desarrollo de diversas líneas de investigación.

En este sentido, muchos han sido los enfoques que se le han dado a los estudios académicos sobre la RSE, y en este sentido, 
Steiner (1972), Davis (1973), Keim (1978) y Shaw \& Post (1993) argumentan que líneas de investigación procedentes de áreas como la teoría económica o la gestión empresarial han sido importantes para ir avanzando en el conocimiento científico de esta. Sin embargo, en palabras de Calveras y Ganuza (2009), no existe mucha literatura sobre la RSE desde la teoría económica, en cambio sí que existen varias reseñas de la literatura académica de la RSE desde el management.

Independientemente de la cantidad de literatura existente desde ambas perspectivas, la reflexión conceptual de la RSE ha puesto un énfasis bastante importante en la economía y el management, por encima, inclusive, de la perspectiva de la sociología y de la política (Lee, 2008). Este hecho se evidencia tanto desde el punto de vista de publicación académica como de autores más citados (según el Social Science Citation Index) en el campo de la RSE.

En cuanto a publicación académica, diversos autores evidencian este hecho: por ejemplo, Locket, Moon \& Visser (2006), en su estudio sobre el estado de la investigación de la RSE en diez años (1992-2002), concluyen que el top 10 de las revistas académicas con mayores publicaciones está conformado, en su orden, por Academy of Management Review; Academy of Management Journal; Strategic Management Journal; Administrative Science Quarterly; Journal of Business Ethics; Harvard Business Review; Business Ethics Quarterly; California Management Review; Journal of Personality and Social Psychology y Organization Science. De estas revistas, nueve (9) pertenecen a la disciplina del management, destacando las dos primeras, cuya edición es de la reconocida Academy of Management (AOM) y solo una (1), la Journal of Personality and Social Psychology, pertenece a una disciplina diferente al management.
Así mismo, los citados autores concluyen que los temas más investigados fueron los relacionados con el medioambiente y la ética, y agregan que la investigación empírica, en su mayoría, ha sido de corte cuantitativo, y la investigación teórica fue principalmente no normativa.

En esta misma línea, Broomhill (2007) argumenta que durante la década pasada hubo un notable aumento de la literatura científica de RSE debido, principalmente, al importante incremento de publicaciones en las business and management journals, lo que se evidencia con los más de 800 artículos científicos identificados en la fuente bibliográfica online ingentaconnect y los 535 artículos encontrados en la reconocida base de datos SCOPUS, publicados en las siguientes revistas académicas: Journal of Business Ethics; Corporate Governance and Social Responsibility; Corporate Social Responsibility and Environmental Management; Journal of Corporate Citizenship; Corporate Governance: International Journal of Business in Society; Business Ethics, a European Review; Business Ethics Quarterly; Business \& Society; Business and Society Review; Journal of Management Studies; Enterprise and Society; Corporate Communications: An International Journal; Journal of Business Strategy; International Journal of the Economics of Business y Stanford Social Innovation Review.

Por su parte, otro grupo de autores (Alvarado, Bigné, Curras y Sánchez, 2011) también muestra la evolución de la literatura científica de RSE desde la perspectiva del management. En concreto, llevaron a cabo una revisión de 35 años de trabajos de investigación (1972-2006) publicados en revistas de $\mathrm{ma}$ nagement indexadas; encontraron un total de 1082 artículos científicos. Este estudio es muy interesante, ya que analiza la evolución epistemológica que la RSE ha tenido desde la literatura del management. 
El interés de la investigación de la RSE en $m a$ nagement también lo evidencia Vaca (2012) en un trabajo más reciente, en el que llama la atención la elaboración de una tabla que muestra una serie de estudios en la materia, realizados entre 1998 y 2007 . Después del respectivo análisis, se concluye que en total fueron publicados 62 artículos científicos, 14 de los cuales fueron de corte teórico y 48 empíricos; de estos últimos, 20 aplicaron la entrevista, 18 el estudio de caso, 8 el análisis de contenido y 1 la encuesta.

En relación con autores más citados (según el Social Science Citation Index) en el campo de la RSE, De Bakker, Groenewegen \& Den Hond (2005) argumentan que el top $10 \mathrm{de}$ los autores más representativos y citados en RSE lo conforman, en su orden, Jones (1995); Wood (1991); Mitchell, Agel \& Wood (1997); Aupperle, Carroll \& Hatfield (1985); McGuire, Sundgren \& Schneeweis (1998); Clarkson (1995); Cochran \& Wodd (1984); Wartick \& Cochran (1985), Klassen \& McLaughlin (1996) y Waddock \& Graves (1997), los cuales han publicado, sin excepción, en revistas de management. Concretamente el primero, el segundo, los terceros, el sexto y los octavos publicaron en la Academy of Management Review; los cuartos, los quintos y los séptimos en la Academy of Management Journal; los novenos en la Management Science y los décimos en la Strategic Management Journal. De manera más específica, se han desarrollado múltiples líneas de investigación desde el ámbito general del management. Al respecto, Mahon \& Griffin (1999) y Walsh, Weber \& Margolis (2003) tienen contundentes conclusiones. Para los primeros, en 25 años de estudios científicos en la materia se han establecido islas de investigación, con señales parciales, acerca de un cuadro enorme por pintar. Para los segundos, en 35 años de investigación se ha incrementado el cuerpo del debate con diferentes perspectivas que han dispersado el conocimiento, oscureciendo con ello el citado cuadro.
Finalmente, teniendo en cuenta estas interesantes conclusiones, y con el objetivo de aportar a la temática en cuestión, a partir de los trabajos de Lockett, Moon \& Visser, (2006); Bigné, Chumpitaz, Andreu \& Swaen, (2010); Pérez (2011) y Perdomo y Escobar (2011), se propone agrupar las líneas actuales de investigación de RSE desde el ámbito general del management en las siguientes cinco (5) categorías: (i) línea RSE - RFE: correlación existente entre la responsabilidad social empresarial y los resultados financieros empresariales; (ii) línea RSE - Organización, enfocada en las características del directivo: análisis de las actitudes, características y remuneración del directivo frente a la RSE; (iii) línea RSE - Marketing: análisis de diversas temáticas relacionadas con estrategias y acciones empresariales en la materia, y la percepción de los consumidores sobre dichas actuaciones; (iv) línea RSE - Accountability: análisis de la rendición de cuentas (transparencia) y el reporting corporativo y (v) línea RSE - Estrategia social y modelo empresarial para crear, compartir valor y contribuir a la sostenibilidad empresa-sociedad.

A continuación, se procederá a mostrar los resultados específicos de la investigación de la RSE desarrollada en el sector hotelero durante los últimos diez años (2004-2014).

\section{Investigación académica de la RSE en el sector hotelero}

La literatura específica sobre RSE en la industria hotelera se puede calificar como escasa si se compara con otros temas de mayor desarrollo investigativo en el sector turístico/ hotelero, y más aún si se contrasta con otros sectores económicos. En este sentido, Bohdanowicz \& Zientara (2009) y McGehee, Wattanakamolchai, Perdue \& Calvert (2009) argumentan que pocas investigaciones han analizado aspectos relacionados con la RSE en el sector hotelero. 
De igual manera, hay pocos trabajos de investigación, desde el punto de vista holístico, sobre la RSE en el ámbito turístico, más concretamente en el sector hotelero (Bohdanowicz, 2007; Holcomb, Upchurch \& Okumus, 2007). En palabras de Martínez, Pérez y Rodríguez del Bosque (2013,p. 143), "a pesar de que gran número de investigadores y profesionales del sector coinciden en que la RSE se ha convertido en una función necesaria, gran parte de la investigación se ha centrado en la relación entre la RSE y los resultados financieros (Lee \& Park, 2009, citado en Martínez, Pérez y Rodríguez del Bosque, 2013), dedicando una menor atención a aclarar cómo se define esta noción, qué papel desempeña en las empresas hoteleras y cómo debe ser implementada en este sector (Bohdanowicz \& Zientara, 2009; Tsai, Hsu, Chen, Lin \& Chen, 2010; Font, Walmsley, Cogotti, McCombes \& Haüsler, 2012). Además, la mayoría de los estudios existentes sobre la práctica de la RSE en este sector se limitan a cuestiones medioambientales, restando importancia a aspectos sociales y culturales (Ayuso, 2006, citado en Rodríguez-Antón, Alonso-Almeida y Celemín-Pedroche, 2013; Bohdanowicz \& Zientara, 2009; Fernández y Cuadrado, 2011)".

Por otra parte, Jiménez y Gessa (2010), citado en Vaca (2012), también evidencian la escasez de literatura científica sobre RSE en el sector hotelero. En su estudio sobre revisión y análisis de la producción científica de la RSE y la gestión hotelera en el periodo 2000-2010, concluyen que del total de artículos analizados solo el 3,1\% prestaba especial atención a la RSE y el 2,6\% a la gestión de la RSE en el sector hotelero. Estos resultados muestran la necesidad de realizar más estudios de la RSE en dicho subsector y su retraso en dicho ámbito de estudio (Vaca, 2013, citado en Martínez, Pérez y Rodríguez del Bosuqe, 2013).

En este sentido, autores como RodríguezAntón, Alonso-Almeida y Celemín-Pedroche
(2013, p. 22) han realizado un esfuerzo interesante por contribuir al entendimiento del contenido de los trabajos de la RSE en el sector hotelero. En palabras de los autores, "existen tres grandes líneas de investigación relacionadas con RSE en el sector hotelero. En la primera, un número elevado de estudios actuales analizan las prácticas de RSE que implementan las compañías hoteleras (por ejemplo: Carlsen et al., 2001; Bohdanowicz, 2006; Ayuso, 2006; Carmona-Moreno et al., 2004; Scanlon, 2007; Erdogan \& Baris, 2007; Kasim, 2007; Cheng \& Parker, 2010). A continuación, una menor atención ha sido dedicada por la literatura al análisis de la publicación de informes de RSE por parte de los hoteles (por ejemplo: Holcomb, Upchurch \& Okumus, 2007; Priego y Palacios, 2008; Bohdanowicz \& Zientara, 2008), constituyendo la segunda línea de investigación. Estos estudios, relacionados con la presentación de informes RSE en la industria hotelera, están mayoritariamente limitados a muestras reducidas (10-15 compañías) o áreas geográficas específicas, pero ninguno de estos estudios da una visión global de los informes de RSE de la industria hotelera (De Grosbois, 2012). El último grupo de estudios publicados en el contexto hotelero viene configurado por aquellos que analizan la relación existente entre las actividades de RSC y su impacto en el rendimiento financiero (por ejemplo: García y Armas, 2007; Lee \& Park, 2009)".

No obstante lo anterior, analizando la descripción que hacen Rodríguez-Antón, AlonsoAlmeida y Celemín-Pedroche (2013) sobre los resultados de los estudios citados en el párrafo anterior, se puede concluir que los de la primera línea son estrictamente de corte medioambiental; en cuanto a los de la segunda línea, lo es uno de ellos (el de Priego y Palacios, 2008) y, finalmente, en relación con los de la tercera línea, el de Lee \& Park (2009), en particular, estudia de manera conjunta los sectores hotelero y de casinos. 
Por lo tanto, para una mayor comprensión del tema del estudio, se hace necesario llevar a cabo una revisión de la literatura que cumpla con dos condiciones: la primera, que los estudios se centren solamente en el sector hotelero; y la segunda, que el tema de la RSE se enfoque desde una perspectiva amplia (económica-social-ambiental), y no exclusivamente desde el ámbito ambiental, tal como ha quedado evidenciado en el párrafo anterior.

También es importante mencionar que los trabajos que han analizado la RSE en otros sectores del turismo, por ejemplo, en restaurantes, bares, casinos, transporte aéreo, incluidos aquellos que mezclan el sector hotelero con algún otro sector, forman parte de la revisión de literatura de la RSE del sector turístico en general, pero no específicamente del sector de interés del presente trabajo, es decir, el hotelero.

De esta manera, el tener un enfoque de revisión de trabajos académicos de RSE desde una perspectiva, aplicados exclusivamente al sector hotelero, hace que se obtenga un análisis preliminar característico propio de la RSE en dicho sector, alejado de algunas conclusiones que generalizan la RSE para todo el sector turístico y que pueden derivarse de aquellos trabajos que investigan, dentro de un mismo estudio, la RSE de diferentes sectores del turismo. Para estos casos, lógicamente, los resultados que se obtienen se pueden generalizar para todo el sector (turismo), pero no se pueden especificar para ningún tipo de empresa en particular (hoteles, restaurantes, casinos, aerolíneas, etc.).

Teniendo en cuenta lo anterior, y con el objetivo de contribuir a la búsqueda y difusión de resultados en materia de RSE en la industria hotelera, se ha realizado una revisión de la literatura de trabajos académicos en el tema, llevados a cabo durante la última década (2004-2014), mediante la cual se han encon- trado cincuenta y dos (52) estudios que han sido agrupados en cuatro (4) grandes líneas de investigación (ver tabla 1), a saber: (i) línea RSE-Prácticas, (ii) línea RSE-Accountability; (iii) línea RSE-Marketing y (iv) línea RSE-RFE.

La primera línea se enfoca en el análisis de las prácticas de RSE que las empresas hoteleras llevan a cabo (incluyendo filantropía/ donaciones), así como en los principales factores que motivan u obstaculizan su implementación; además, estudia la influencia del entorno local (economía, política, cultura, desarrollo) en los niveles aplicación de la RSE. La segunda línea orienta sus estudios hacia la rendición de cuentas (transparencia) y el reporting corporativo. La tercera estudia diversas temáticas relacionadas con estrategias y acciones empresariales de marketing en RSE, así como la relación y percepción de los consumidores y clientes internos (empleados) sobre las actuaciones en la materia. La cuarta analiza la correlación existente entre la RSE y los resultados financieros empresariales.

A continuación, se describen los resultados más importantes para cada una.

\section{RSE-Prácticas}

El estudio de Do Céu Almeida (2005), enfocado en hoteles 4 y 5 estrellas de Portugal, revela que, aunque existen implementaciones aisladas de acciones en RSE, la gran mayoría de las empresas del estudio parecen no haber asumido el concepto.

Kasim (2006) analiza la relevancia y aplicación de lo que la autora denomina BESR (The Business Environmental and Social Responsibility) en el sector hotelero, y llega a la conclusión de que el sector tiene una directa y estrecha relación con su entorno físico y social, que ha generado repercusiones negativas en este último, que deben ser mitigadas, lo cual es importante, no solo para el bien del 
Tabla 1. Líneas de investigación de RSE en el sector hotelero 2004-2014

\begin{tabular}{|c|c|c|c|}
\hline $\begin{array}{l}\text { Línea de } \\
\text { investigación }\end{array}$ & Descripción & Autores & $\begin{array}{l}\text { Número de } \\
\text { estudios }\end{array}$ \\
\hline $\begin{array}{l}\text { RSE - } \\
\text { Prácticas }\end{array}$ & $\begin{array}{l}\text { Análisis de las prácticas en } \\
\text { RSE que llevan a cabo las } \\
\text { empresas hoteleras (incluyendo } \\
\text { filantropía / donaciones), así } \\
\text { como los principales factores } \\
\text { que motivan u obstaculizan } \\
\text { su implementación; además, } \\
\text { estudia la influencia que tiene } \\
\text { el entorno local (economía, } \\
\text { política, cultura, desarrollo) en } \\
\text { los niveles aplicación de la RSE. }\end{array}$ & $\begin{array}{l}\text { Do Céu Almeida (2005); Kasim (2006); Nord (2006); } \\
\text { Palau (2006); PricewaterhouseCoopers (2006), } \\
\text { citados en Tepelos (2008); McGehee \& Wattanaka- } \\
\text { molchai (2007); Font (2008); McGehee et al. (2009); } \\
\text { Tsai et al. (2010); Cardona y Hernández (2011); } \\
\text { Fernández y Cuadrado (2011); Huimin \& Ryan } \\
\text { (2011); Levy \& Park (2011); Calveras (2012); Garay } \\
\text { y Font (2012); Mattera y Moreno (2012); Melo, Cox } \\
\text { Moura-Leite \& Carlton Padgett (2012); Peña y Serra } \\
\text { (2012b); Vaca (2012); Eme, Obal, Inyang \& Effiong } \\
\text { (2013); Garay y Font (2013); Huimin, Ryan, Li \& } \\
\text { Gao (2013); Khunon \& Muangasame (2013); Gon- } \\
\text { zález Jiménez de la Espada (2013); Martínez, Pérez } \\
\text { y Rodríguez del Bosque (2013); Rodríguez-Antón, } \\
\text { Alonso-Almeida y Celemín-Pedroche (2013); Ferus- } \\
\text { Comelo (2014); Sánchez-Fernández, Vargas Sánchez } \\
\text { y Remoaldo (2014); Sandve y Øgaard (2014); Sepúl- } \\
\text { veda, Ordóñez y Prada (2014). }\end{array}$ & 31 \\
\hline $\begin{array}{l}\text { RSE - } \\
\text { Marketing }\end{array}$ & $\begin{array}{l}\text { Análisis de diversas temáticas } \\
\text { relacionadas con estrategias y } \\
\text { acciones empresariales en la } \\
\text { materia, así como la relación y } \\
\text { percepción de los consumidores } \\
\text { y clientes internos (empleados) } \\
\text { sobre dichas actuaciones. }\end{array}$ & $\begin{array}{l}\text { Kasim (2004); Deery, Jago \& Stewart (2007); Mac- } \\
\text { kenzie \& Peters (2012); Tsai, Tsang \& Cheng (2012); } \\
\text { Aminudin (2013); Kucukusta, Mak \& Chan (2013); } \\
\text { Martínez y Rodríguez del Bosque (2013); Benavides- } \\
\text { Velasco, Quintana-García y Marchante-Lara (2014); } \\
\text { Fu, Ye \& Law (2014); Gao \& Mattila (2014); Trías y } \\
\text { García (2014). }\end{array}$ & 11 \\
\hline $\begin{array}{l}\mathrm{RSE}-A c- \\
\text { countability }\end{array}$ & $\begin{array}{l}\text { Análisis de la rendición de } \\
\text { cuentas (transparencia) y } \\
\text { reporting corporativo. }\end{array}$ & $\begin{array}{l}\text { Bohdanowicz (2007); Henderson (2007); Holcomb, } \\
\text { Upchurch \& Okumus (2007); Bohdanowicz \& Zien- } \\
\text { tara (2008 y 2009); Martos (2011); Rey-Maquieira, } \\
\text { Aquiló, González, Ramos y Razumaba (2011); De } \\
\text { Grosbois (2012); Font et al. (2012); Dzingai (2013). }\end{array}$ & 10 \\
\hline RSE - RFE & $\begin{array}{l}\text { Correlación existente entre la } \\
\text { RSE y los resultados financieros } \\
\text { empresariales. }\end{array}$ & García y Del Mar (2007). & 1 \\
\hline
\end{tabular}

Fuente: Elaboración propia.

entorno, sino también para la sostenibilidad de la propia industria.

En el trabajo de Nord (2006) se realiza un análisis de la incidencia que la cultura y el entorno local tienen sobre los niveles de RSE de las empresas de alojamiento, mediante un benchmarking de 65 hoteles pertenecientes a los grupos internacionales Rezidor Hotel
Group (24 Radisson S. A. S. Hotels y 6 Park Inns) e InterContinental Hotels Group (8 Crowne Plazas y 27 Holiday), ubicados en Suecia, Noruega, Dinamarca, Islandia, el Reino Unido, Arabia Saudita, Omán, Egipto, Baréin y los Emiratos Árabes Unidos. Se concluye que los sistemas políticos y el nivel de desarrollo económico de una región afectan los niveles de la RSE. 
Por otra parte, Palau (2006), citado en Tepelus (2008), lleva a cabo un estudio en 24 cadenas hoteleras que representan el $60 \%$ de la oferta hotelera de Catalunya (España). El autor concluye que la mayoría de estas compañías no desarrolla estrategias de RSE debido a la falta de conocimiento en el tema y en estándares internacionales, tales como el GRI, SA8000, entre otros.

El estudio PricewaterhouseCoopers (2006), citado en Tepelus (2008), enfocado en el top 14 de las grandes cadenas hoteleras europeas, concluye que, a pesar de algunos recientes progresos, particularmente en términos de implementación de políticas medioambientales, el sector está por detrás de otras industrias europeas en materia de estrategias de RSE.

McGehee \& Wattanakamolchai (2007) estudian la RSE en la industria hotelera en los Estados Unidos, tanto en el ámbito corporativo como en el de propiedad, y concluyen que casi todos los hoteles han hecho contribuciones de habitaciones, dinero en efectivo, certificados de regalo, entre otros, mientras que poco más de la mitad ha realizado un seguimiento de las acciones de contribuciones/voluntariado a la comunidad.

Font (2008) utiliza la metodología Action Research para analizar el comportamiento de 43 hoteles de Colombia respecto a su RSE, en el sentido de contribuir al alivio de la pobreza en el país. El autor concluye que la acción de compra a proveedores locales es una de las actividades más utilizadas y la económicamente más rentable para promover la erradicación de la pobreza.

McGehee et al. (2009) llevan a cabo un análisis cuantitativo de las donaciones corporativas realizadas a la comunidad por parte del sector hotelero de los Estados Unidos, siendo el valor obtenido de más de 815 millones dólares, lo que representa el 3,6\% de los beneficios totales de la industria en
2005. Este estudio es importante porque ha sido el primero en evidenciar el valor real en dinero de las contribuciones de la industria hotelera.

Tsai et al. (2010) aplican al Far Eastern Plaza Hotel - un prestigioso hotel internacional de Taipéi (Taiwán, China) operado por The Shangri-La Hotels and Resorts, el grupo hotelero asiático más grande del mundo- un enfoque integrado que utiliza programas complejos de relaciones estructurales para ayudar a los administradores de hoteles turísticos internacionales a seleccionar los programas de RSE menos costosos y más adecuados para el cumplimiento del doble objetivo empresa-sociedad, es decir, crear ventajas competitivas para el hotel, al tiempo que se beneficia a la sociedad más cercana (comunidad de influencia).

Cardona y Hernández (2011) realizan un estudio en diez hoteles PYME de la ciudad de Cartagena (Colombia), con el objetivo de indagar acerca de las acciones de la empresa en temas de RSE e identificar la percepción de los gerentes sobre esta. La principal conclusión ha sido que los gerentes confunden la idea de RSE con filantropía o donaciones y ayudas sociales.

En otro trabajo, Fernández y Cuadrado (2011) realizan una revisión de la literatura sobre estudios de la RSE en el sector hotelero, argumentando que los estudios de la RSE en el ámbito hotelero se han dirigido hacia el conocimiento de la visión de la RSE, la adopción de políticas de RSE, el impacto económico de la RSE y la comunicación del comportamiento socialmente responsable.

El estudio de Huimin \& Ryan (2011) se basa en la opinión de 257 directores hoteleros de China, quienes concluyen que la aplicación de principios de RSE en sus establecimientos se facilita cuando la administración mantiene una fuerte postura ética. 
Levy \& Park (2011) identifican y analizan las prácticas de RSE de los hoteles ejecutivos de Washington y San Francisco, en los Estados Unidos, y sus beneficios. Concluyen que las actividades más realizadas son las ambientales enfocadas en energía y la gestión del agua y residuos.

Calveras (2012) hace un estudio de la RSE interna (prácticas de recursos humanos) y externa (temas sociales y ambientales) de los hoteles de las Islas Baleares de España, y demuestra que estos dos tipos de RSE, a priori no relacionadas, sí que son complementarias y están ligadas a la calidad del producto.

Garay \& Font (2012), en un estudio dirigido a las PYME de alojamiento de Cataluya, analizan las prácticas de RSE y las razones que llevan a dichas empresas a aplicarlas. Concluyen que las principales motivaciones de los directores para la implantación de acciones de RSE han sido, en su orden, las razones altruistas (protección medioambiental, estilo de vida y compromiso social), económicas/competitivas (reducción de costos y mejora de la imagen) y la legitimación ante sus stakeholders.

Mattera y Moreno (2012) llevan a cabo un análisis comparativo entre la RSE de NH Hoteles y Meliá Hotels Internacional. Argumentan que estas empresas ya tienen implicaciones estratégicas, incorporando acciones significativas cuando se enfocan en la reducción de los daños ambientales, como la del consumo de energía mediante las nuevas tecnologías, el uso de materiales reciclados y la compra de productos de comercio justo.

Melo, Cox Moura-Leite \& Carlton Padgett (2012) identifican en su trabajo lo que cuatro hoteles de lujo (4 y 5 estrellas) en Natal (Río Grande del Norte, Brasil), mediante los responsables de RSE (3 gerentes propietarios, 1 gerente y 1 responsable de recursos humanos) entienden por RSE y la forma en que la ponen en práctica en sus negocios. Los autores encuentran que existe un evidente interés en el tema, pero queda mucho por hacer para el fomento de un impacto social positivo.

Peña y Serra (2012b) llevan a cabo un estudio de caso en la empresa Park Hotel de la ciudad de Santa Marta (Colombia); proponen una metodología para la realización de un diagnóstico y un plan de mejoramiento en RSE aplicable a los establecimientos hoteleros, argumentando que el hotel del estudio se encuentra más cerca de la proactividad que de la reactividad legal, ya que comienza a llevar a cabo prácticas de RSE a las que no está obligada a realizar por ley. Los autores proponen, además, un plan de mejoramiento que comprende 20 acciones de RSE en 9 áreas de gestión del hotel vinculadas directamente con sus stakeholders y relacionadas con la RSE, tanto en el ámbito interno como externo de la organización.

Vaca (2012) analiza los factores determinantes del nivel de desarrollo de la RSE de las cadenas hoteleras españolas y sus consecuencias. Concluye, por una parte, que la RSE está asociada al cumplimiento de la ley, la satisfacción de sus stakeholders y la creación de valor para estos; por otra parte, determina que los factores motivadores identificados para el desarrollo de la RSE son la competitividad, la presión de los stakeholders y la ética, siendo más representativo el segundo.

La investigación de Eme et al. (2013) revela que las prácticas de RSE de los hoteles PYME de Nigeria son mínimas y se dan en un nivel informal, por lo que recomiendan que sean integradas al funcionamiento empresarial, lo que podría acelerar el desarrollo de todo el país.

A partir de una muestra de cerca de 400 hoteles PYME de Catalunya (España), Garay \& Font (2013) demuestran que, además de las variables tradicionales del negocio, se 
deberían tener en cuenta el estilo de vida de los propietarios/gerentes y la legitimidad social ante los stakeholders como motivaciones fundamentales para el comportamiento responsable.

El trabajo de Huimin et al. (2013) examina la existencia de una posible relación entre las conexiones políticas de gerentes de hoteles chinos y la adopción de políticas de RSE en la industria hotelera china. El estudio concluye que aquellos hoteles con mejores conexiones políticas (en donde se reglamentan las normativas en materia de RSE) pueden mostrar evidencias de altos niveles de conciencia y adopción de políticas de RSE.

Khunon \& Muangasame (2013) llevan a cabo un estudio de caso en Tailandia, que evidencia las diferencias y similitudes entre la gestión de la RSE de una cadena hotelera internacional (The Banyan Tree Bangkok) y un hotel local (The Chumphon Cabana Resort). La investigación revela que las motivaciones internas son la base del éxito de la RSE del hotel local, mientras que, para la cadena internacional, este se relaciona con el nivel de la firma.

González Jiménez de la Espada (2013), en su estudio sobre el sector hotelero español, argumenta que los procesos tradicionales de integración vertical deben migrar hacia procesos de integración horizontal, pues estos constituyen una acción concreta de RSE que permite afrontar en mejores condiciones los cambios estructurales que el sector necesita.

El estudio de caso de Martínez, Pérez y Rodríguez del Bosque (2013), centrado en la RSE de Meliá Hotels International, aporta información relevante sobre aspectos relativos a la definición de este concepto, el nivel de conocimiento por parte de la empresa, sus preocupaciones en materia de RSE, el tipo de iniciativas socialmente responsables desarrolladas y el nivel de participación de la compañía en dichas actividades.
El trabajo de Rodríguez-Antón, Alonso-Almeida y Celemín-Pedroche (2013) concluye que, a pesar de la crisis económico-financiera que ha afectado al sector, tres (3) de las principales cadenas hoteleras españolas están apostando por mantener y potenciar las actuaciones de RSE, hasta el punto de convertirlas en estratégicas. En concreto, las cadenas hoteleras NH y Meliá Hotels International se han enfocado en la defensa del medio ambiente y en la reducción y más eficiente uso del consumo energético, entre otras actuaciones. Por otra parte, Confortel ha concentrado sus esfuerzos en lograr una accesibilidad universal en todos los hoteles del grupo.

La investigación de Ferus-Comelo (2014) estudia el impacto de las acciones de RSE de dos (2) hoteles cinco (5) estrellas (Vivanta by Taj Hotels and Resorts y Leela Kempinski Hotel) de Goa, India. El estudio concluye que la RSE en la industria del turismo, especialmente en el sector hotelero de la India, actualmente se limita al auto-reporting corporativo sobre los indicadores que eligen las empresas y, en algunos casos, al mínimo requerido por los cuerpos reguladores del Estado.

Sánchez-Fernández, Vargas Sánchez y Remoaldo (2014) estudian la relación entre la teoría institucional y la RSE de los hoteles de tres (3), cuatro (4) y cinco (5) estrellas de Galicia (España) y la región norte de Portugal. Los autores concluyen que los hoteles están enmarcados dentro de un contexto institucional caracterizado por el cumplimiento y las presiones regulatorias en esta materia.

Sandve \& Øgaard (2014) evidencian que la obligación ética y las normas subjetivas influyen en la decisión de llevar a cabo comportamientos socialmente responsables en los gerentes de hoteles PYME de Noruega.

Por último, Sepúlveda, Ordóñez y Prada (2014) evalúan el perfil de las acciones de 
gestión que se relacionan con los conceptos de RSE del sector hotelero de la ciudad de Bucaramanga (Colombia) a partir del análisis de sus prácticas y operaciones en la materia. La evaluación permite concluir que la RSE, como estrategia de gestión, es comprendida como un modelo market pull que enfoca sus principales beneficios en el posicionamiento de las organizaciones; no obstante, la RSE no es vista como un elemento estratégico de inversión, sino como un gasto adicional al proceso de direccionamiento organizacional.

\section{RSE-Accountability}

Bohdanowicz (2007) realiza un análisis del recurso HER (Hilton Environmental Reporting) en el Hilton International. Se trata de un sistema de información ambiental y benchmarking, que destaca una serie de aspectos que deben abordarse al desarrollar este tipo de sistemas. El análisis concluye que puede ser muy útil para otros negocios turísticos y hoteleros en su intento por desarrollar su propia información, seguimiento y evaluación comparativa de sus planes de RSE.

En su estudio, Henderson (2007) analiza las reacciones que las empresas hoteleras en Phuket (Tailandia) reportaron en sus informes de RSE después del desastre generado por el tsunami, y concluye que las respuestas de los hoteleros muestran cierto nivel de compromiso con la RSE; sin embargo, se evidencian algunas tensiones entre las actividades filantrópicas y las comerciales.

Holcomb, Upchurch \& Okumus (2007) utilizan el método del análisis de contenido en las páginas web, los reportes anuales y los reportes de RSE de las diez cadenas hoteleras más importantes del mundo, según la prestigiosa revista Hotels Magazine, para identificar y describir las actividades de RSE que ellas realizan. Concluyen que el $80 \%$ reporta acciones relacionadas con donaciones, el $60 \%$ registra políticas de RSE y el $40 \%$ menciona la RSE en su misión o visión.

Bohdanowicz \& Zientara (2008) desarrollan un estudio de caso en una de las cadenas hoteleras más grandes de Escandinava: Scandic. En concreto, llevan a cabo un análisis de los informes de RSE y otros documentos internos, complementado con entrevistas a directores de hotel, y concluyen que las implicaciones en los temas de la RSE de sus establecimientos tienen unos efectos positivos en la administración basada en el recurso humano, así como también en el apoyo a la comunidad local y en la promoción de la sostenibilidad medioambiental.

Bohdanowicz \& Zientara (2009) realizan otro trabajo que consiste en analizar el contenido de los informes de RSE de los diez grupos hoteleros más grandes del mundo (InterContinental Hotels Group, Wyndham, Marriott International, Hilton Hotels Corporation, Accor International, Choice Hotels International, Best Western, Starwood Hotels \& Resorts Worldwide, Carlson Hospitality y Global Hyatt). Los investigadores concluyen que diversas acciones en la materia han contribuido a mejorar la calidad de vida de las comunidades locales, así como también el bienestar de los empleados; no obstante, sugieren que las cadenas hoteleras internacionales pueden jugar un rol más importante en el desarrollo de las comunidades anfitrionas.

Martos (2011) hace un análisis de los informes de RSE de la NH Hoteles, Sol Meliá y Paradores de Turismo de España, y posteriormente un estudio in situ, para concluir que a las empresas del estudio les resulta rentable atender a sus stakeholders (clientes y empleados prioritariamente) bajo criterios de RSE, concibiendo además como una inversión la protección de los atractivos turísticos de los lugares donde operan. 
La investigación de Rey-Maquieira et al. (2011) propone una metodología para identificar si las empresas de alojamiento utilizan la RSE como un recurso estratégico para el negocio; para ello, llevan a cabo un análisis de los informes de RSE o información sobre esta en las webs de las 25 cadenas hoteleras más grandes del mundo. Los autores concluyen que la RSE en las cadenas hoteleras tiene generalmente un carácter filantrópico y reactivo, pero no estratégico.

En el estudio realizado por De Grosbois (2012) se analizan los informes de RSE de las 150 primeras empresas hoteleras más grandes del mundo; se encuentra que mientras un número grande de hoteles reporta sus metas en RSE, un número mucho más pequeño provee detalles de las iniciativas específicas y del funcionamiento real alcanzado en la materia.

En el trabajo de Font et al. (2012) se lleva a cabo un benchmarking de las políticas y prácticas de RSE del top 10 de los grupos hoteleros europeos a partir del reporting generado por aquellas e información pública encontrada en sus sitios web, lo cual fue complementado con la aplicación de un cuestionario a los responsables de RSE. Como resultado se encuentra que, de manera general, los sistemas corporativos no se reflejan en las operaciones, el desempeño ambiental se basa en el ecoahorro, los objetivos de las políticas laborales se enfocan en cumplir con la legislación local, las políticas socioeconómicas son hacia adentro y no tienen un impacto en el destino, $y$ el compromiso con la clientela es limitado.

Por último, Dzingai (2013) analiza las actividades que realizan en RSE los grupos hoteleros de Zimbabwe, mediante un análisis de contenido de sitios web, informes anuales y declaraciones en la misión. El análisis concluye que las empresas dan más importancia al funcionamiento financiero que a los temas sociales y ambientales, y si se comparan con cadenas hoteleras de otros países en vía de desarrollo, en materia de reporting se quedan atrás.

\section{RSE-Marketing}

La investigación de Kasim (2004) evidencia la propensión de los turistas a elegir los hoteles de Pulau Pinang (Malasia) con base en criterios ambientales y sociales. El estudio concluye que la inclinación a los atributos responsables (cultura local, cocina local y personal experimentado, feliz y amigable) parece depender de qué tan relevantes son dichos atributos para la calidad de la experiencia de sus vacaciones.

Deery, Jago \& Stewart (2007) estudian la percepción sobre la RSE de los empleados de un hotel 5 estrellas en Australia, y concluyen que, si bien el hotel parece tener un bajo nivel de rotación de personal, existe una insatisfacción relacionada con la seguridad del empleo, el nivel de compromiso desde el hotel a los empleados y el acceso a equipos adecuados para hacer su trabajo correctamente.

Mackenzie \& Peters (2012) investigan cómo los gerentes de talento humano de hoteles de media y alta categoría de Hong Kong tratan de influir en el comportamiento de sus empleados en materia de RSE, tanto en las propias empresas como en la comunidad. Se determina que los gerentes del estudio difieren en los estilos de liderazgo, lo que influye en la percepción de sus empleados del papel de la RSE en la industria y la comunidad.

El estudio de Tsai, Tsang \& Cheng (2012) examina la importancia percibida de diversos atributos de la RSE por parte de empleados de los hoteles de Hong Kong de acuerdo con su real funcionamiento. Los resultados del análisis muestran que los hoteles de Hong Kong funcionan bien en 17 de los 30 atributos de la RSE y, en concreto, el atributo "promueve la innovación en productos y los servicios" 
es el mejor valorado, en tanto que el atributo "demuestra un compromiso con el medio ambiente" requiere de una atención inmediata.

El trabajo de Aminudin (2013) identifica la relación entre la RSE de los hoteles "verdes" de Malasia y la identificación organizacional mediante sus empleados, evidenciando que las responsabilidades de estos no solo se enfocan en las funciones de su trabajo, sino que tienen en cuenta la relación con los stakeholders, proveedores, comunidad, clientes y medio ambiente.

Kucukusta, Mak \& Chan (2013) evidencian en su trabajo la percepción de los turistas de Hong Kong sobre la RSE de sus hoteles de cuatro (4) y cinco (5) estrellas. Los visitantes identificaron cinco (5) factores de RSE: comunidad, política, misión y visión, lugar de trabajo y medio ambiente; de estos, el medio ambiente y la misión y visión son los que más se valoran en cuanto a sus preferencias para quedarse, la disponibilidad de pagar, la percepción de la calidad del servicio y la imagen corporativa.

En otra investigación, Martínez y Rodríguez del Bosque (2013) concluyen que la RSE se ha vuelto crucial para las empresas hoteleras debido a la influencia que puede tener en la lealtad de los consumidores. En este sentido, argumentan que dicha lealtad se ve afectada indirectamente por la RSE de los hoteles españoles mediante el rol que juega sobre la confianza, la identificación del cliente con la compañía y la satisfacción.

Benavides-Velasco, Quintana-García y Marchante-Lara (2014) analizan cómo la práctica del TQM (Total Quality Management) y la RSE influyen en los resultados del negocio con sus stakeholders, principalmente empleados y clientes, en el sector hotelero de Andalucía (España). Los autores concluyen que la adopción de medidas de TQM y RSE mejora la capacidad de los hoteles para crear beneficios para sus stakeholders, y que estos resultados tienen un efecto positivo sobre el funcionamiento de los hoteles.

El estudio de Fu, Ye \& Law (2014) muestra evidencias sobre la influencia de la RSE de los hoteles en China sobre el comportamiento de la ciudadanía organizacional, mediante los efectos de la identificación organizacional y el compromiso. Se revelan relaciones entre la percepción de los empleados en materia de RSE y el impacto en su comportamiento.

Gao \& Mattila (2014) examinan la relación entre la satisfacción del cliente y las iniciativas de RSE en hoteles verdes de los Estados Unidos. En concreto, estudian los efectos que tienen en la percepción del huésped sobre la calidez y la competencia de las empresas hoteleras, utilizando mecanismos psicológicos que explican las reacciones de los consumidores. Los resultados muestran que cuando la prestación del servicio es exitosa, la satisfacción del cliente es más alta en hoteles verdes con actividades en RSE, que en aquellos que no las realizan (hoteles no verdes).

Finalmente, el trabajo de Trías y García (2014) se centra en el sujeto de la RSE en el marketing sostenible como un elemento clave para diseñar el marketing interno estratégico de la RSE en los hoteles de lujo de las Islas Baleares de España.

\section{$R S E-R F E$}

García y Del Mar (2007) estudian la incidencia de la RSE (definida por los autores como responsabilidad social-medioambiental) en el rendimiento económico de la empresa hotelera española, concretamente en 80 hoteles de 3, 4 y 5 estrellas de Baleares, Catalunya, Andalucía, Canarias, Comunidad Valenciana y Madrid. Los investigadores concluyen que unos mayores niveles de RSE provocan incrementos de los niveles de rentabilidad, no obstante, recomiendan llevar a cabo un estudio similar 
con empresas hoteleras que presenten menores niveles de beneficios a las del estudio.

\section{Conclusiones}

Después de identificar, analizar y clasificar las diferentes líneas de investigación más importantes de la RSE desde el ámbito general del management, es conveniente hacer una reflexión sobre la evolución o cambio que la RSE ha tenido como campo científico de estudio.

$\mathrm{Al}$ respecto, se pueden distinguir tres tipos de evolución posibles; (i) progresiva, la cual estipula que la literatura ha evolucionado desde la imprecisión o clarificación de constructos centrales y sus relaciones; (ii) heterogénea, en la que el progreso en la literatura sobre RSE ha sido frenado por la continua introducción de nuevos conceptos; y (iii) normativa, que establece que no se ha producido prácticamente ningún tipo de evolución debido al carácter normativo inherente a este tipo de literatura (De Bakker, Groenewegen \& Den Hond, 2005).

Es así como la visión progresiva es la que más se ajusta a la evolución de la literatura de RSE en manangement, pues es evidente que la aparición de nuevos conceptos y nuevas relaciones a partir de la contrastación y la confirmación de trabajos ya existentes no ha frenado la investigación en RSE, sino que la ha fortalecido cualitativa y cuantitativamente, 10 que anula la postura de heterogeneidad. Por otro lado, la idea de ausencia de evolución que subyace de lo normativo se contradice si se analiza la propia evolución que el concepto RSE ha tenido al pasar de una concepción filantrópica y de caridad a una concepción estratégica y de vínculo directo con el desarrollo de la sociedad.

Lo anterior se refuerza si se analiza la evolución del enfoque teórico-metodológico utilizado en las investigaciones de RSE en management. Al respecto, se puede concluir que el enfoque más utilizado en los últimos tiempos se ha caracterizado más por el sentido positivo de la investigación que por el normativo, pues los trabajos han estado más cercanos a la descripción y a la prescripción de la práctica empresarial de la RSE en el sentido del debe ser (best practices) que a la búsqueda de sistemas que predigan el ser de la RSE.

En cuanto a la investigación de la RSE en el sector hotelero, se concluye que sigue siendo escasa, ya que han sido publicados 52 artículos en 10 años (2004-2014), lo que equivale a 5,2 artículos por año, una cifra bastante insípida para un tema tan relevante en nuestros tiempos, como lo es la RSE, y más aún dentro de un subsector como el hotelero, el más importante del sector turismo, que, a su vez, es considerado uno de los sectores económicos más importantes en el mundo.

Del análisis realizado en cada uno de los trabajos se puede concluir que las investigaciones han ido dirigidas al análisis de cuatro áreas: las prácticas de RSE, el accountability, el marketing y la relación con los resultados financieros.

En este sentido, ha habido un predominio de los estudios que se enfocan en la línea de investigación RSE-Prácticas, con 30 trabajos ( $58 \%$ del total), seguidos por las investigaciones en las líneas de RSE-Marketing y RSEAccountability, con 11 (21\% del total) y 10 estudios (19\% del total) respectivamente, $\mathrm{y}$, por último, la línea RSE-RFE, con 1 estudio (2\% del total).

Teniendo en cuenta lo anterior, de manera más específica se recomienda que sigan desarrollándose investigaciones en las cuatro líneas de investigación encontradas, pero haciendo comparaciones y analizando las particularidades, similitudes y diferencias 
entre los hoteles, siendo el tamaño una de las más relevantes, pues es interesante tratar de identificar no solo las buenas prácticas en las diferentes líneas de investigación, sino las principales motivaciones y los obstáculos en torno a la aplicación de la RSE, ya que lo que realmente interesa para las empresas del sector hotelero y la sociedad anfitriona que las acoge es que pongan en acción la RSE de una manera más decisiva, sin importar el tamaño que ostente cada una de ellas.

Por otra parte, es importante recordar que la forma de entender y aplicar la RSE varía de localidad a localidad, de industria a industria y a través del tiempo (Welford, Man \& Chan, 2007, citado en Guédez, 2010), por lo tanto, la RSE aplicada de la misma manera a todos los contextos entorpecería la operatividad de su concepto, medición e implementación (Driver, 2006).

Esta es una justificación científica válida adicional que hace que la presente investigación adquiera una mayor importancia y validez. Sin duda, los resultados mostrados en materia de investigación de RSE en el sector hotelero durante los últimos 10 años, más el detalle de sus principales conclusiones, son un claro aporte para el área de conocimiento y sector empresarial que se está estudiando; y de manera más particular, también son de gran utilidad para ir comprendiendo, cada vez más, la forma en que el sector hotelero ha venido integrando el concepto de la RSE a la práctica organizacional.

No obstante, tal como lo argumenta Robledo (2004), la regla general ante cualquier situación económica, social, ambiental, cultural e inclusive política que se pueda presentar en un entorno geográfico, sea cual sea, es que se debe partir del supuesto de que las responsabilidades sociales son universales; y así como los mercados y las finanzas se han globalizado, deben hacerlo también los elementos básicos que constituyen dichas responsabilidades, es decir, los principios e intereses sociales universalmente aceptados, tales como los derechos humanos, los estándares laborales, el medio ambiente, el desarrollo sostenible, la justicia, entre otros, de tal manera que la empresa tenga una implicación más directa con el bienestar y el desarrollo de la sociedad, y además contribuya a la construcción de un mundo más justo y equitativo para todos.

Sin duda alguna, el sector hotelero tiene el potencial para contribuir a la construcción de una sociedad mejor y de ese mundo más justo y equitativo que tanto se anhela, pero solo será posible si las empresas turísticas hoteleras que forman parte de él deciden incorporar, tanto en la estrategia como en la operación, ese concepto que se conoce como responsabilidad social empresarial, del que hoy en día muchas empresas hablan, pero que pocas practican.

\section{Bibliografía}

Alvarado, A., Bigné, E., Currás, R., \& Sánchez, I. (2011). Epistemological evolution of corporate social responsibility in management: An empirical analysis of 35 years of research. African Journal of Business Management, 5(6), 2055-2064. DOI: 10.5897/AJBM09.136

Aminudin, M. (2013). Corporate social responsibility and employee retention of 'Green' Hotels. Procedia - Social and Behavioral Sciences, 105, 763-771. DOI: 10.1016/j.sbspro.2013.11.079

Benavides-Velasco, C., Quintana-García, C. y Marchante-Larab, M. (2014). Total quality management, corporate social responsibility and performance in the hotel industry. International Journal of Hospitality Management, 41, 77-87. DOI: 10.1016/j.ijhm.2014.05.003

Bigné, E., Chumpitaz, R., Andreu, L., \& Swaen, V. (2005). Percepción de la responsabilidad social corporativa: un análisis cross-cultural. Universia 
Business Review, 5, 14-27. Recuperado de http:// www.redalyc.org/articulo.oa?id=43300503

Bohdanowicz, P. (2007). A case study of Hilton Environmental Reporting as a tool of Corporate Social Responsibility. Tourism Review International, 11(2), 115-131. DOI: $10.3727 / 154427207783948937$

Bohdanowicz, P., \& Zientara, P. (2009). Hotel companies' contribution to improving the quality of life of local communities and the well-being of their employees. Tourism and Hospitality Research, 9(2), 147-158. DOI: 10.1057/thr.2008.46

Bowen, H. (1953). Social Responsabilities of the Businessman. New York: Harper and Brothers.

Broomhill, R. (2007). Corporate Social Responsibility: Key Issues and Debates. Dunstan Paper, 1, 1-60. Recuperado de http://www.dunstan. org.au/resources/publications/Dunstan_Papers_ No_1_2007.pdf

Calveras, A. (2012). External and internal corporate social responsibility: complements through product quality. Evidence from the hotel industry. Recuperado de http://goo.gl/zhrxKl

Calveras, A. y Ganuza, J. (2009). Responsabilidad social corporativa. Una visión desde la teoría económica. Cuadernos Económicos de ICE, 76, 101-118. Recuperado de http://goo.gl/QMeJA2

Cardona, D. y Hernández, J. (2011). La responsabilidad social empresarial desde la perspectiva de los gerentes de los hoteles pymes de la ciudad de Cartagena. Saber, Ciencia y Libertad, 6(11), 91-104.

Carroll, A. B. (1979). A three-dimensional conceptual model of corporate performance. Academy of Management Review, 4(4), 497-505. Recuperado de http://goo.gl/zh3QWP

Creswell, J. W. (2002). Educational research: Planning, conducting, and evaluating quantitative and qualitative research. Upper Saddle River, N. J.: Merrill.

Davis, K. (1973). The case for and against business assumption of social responsibilities. Academy of Management Journal, 16(2), 312-322. Recuperado de http://goo.gl/esLJKW

De Bakker, F. G. A., Groenewegen, P., \& Den Hond, F. (2005). A bibliometric analysis of 30 years of research and theory on corporate social responsibility and corporate social performance. Business \& Society, 44(3), 283-317. DOI: $10.1177 / 0007650305278086$

De Grosbois, D. (2012). Corporate social responsibility reporting by the global hotel industry: Commitment, initiatives and performance. International Journal of Hospitality Management, 31(3), 896-905. DOI: 10.1016/j.ijhm.2011.10.008

Deery, M., Jago, L., \& Stewart, M. (2007). Corporate Social Responsibility within the Hospitality Industry. Tourism Review International, 11(2), 107-114.

Do Céu Almeida, M. (2005). La empresa socialmente responsable: estudio empírico en el sector hotelero. Revista Iberoamericana de Contabilidad de Gestión, 5, 45-72. Recuperado de http://goo. $\mathrm{gl} / \mathrm{er} 8 \mathrm{i0f}$

Driver, M. (2006). Beyond the stalemate of economics versus ethics: corporate social responsibility and the discourse of the organizational self. Journal of Business Ethics, 66(4), 337-356. Recuperado de http://goo.gl/GgQ1D1

Dzingai, K. N. (2013). CSR reporting among Zimbabwe's hotel groups: A content analysis. International Journal of Contemporary Hospitality Management, 25(4), 595-613. DOI: 10.1108/09596111311322943

Eme, J.E., Obal, U.E. U., Inyang, O. I., \& Effiong, C. (2013). Corporate social responsibility in small and medium scale enterprises in Nigeria: An exam- 
ple from the hotel industry. International Journal of Business and Management, 8(14), 119-126. DOI: 10.5539/ijbm.v8n14p119

Fernández, M.y Cuadrado, R. (2011). La responsabilidad social empresarial en el sector hotelero. Revisión de la literatura científica. Cuadernos de Turismo, 28, 47-57. Recuperado de http://www. redalyc.org/articulo.oa? $\mathrm{id}=39821278003$

Ferus-Comelo, A. (2014). CSR as corporate selfreporting in India's tourism industry. Social Responsibility Journal, 10(1), 53-67. DOI: 10.1108/ SRJ-11-2012-0144

Font, X. (2008). Sostenibilidad y alivio de la pobreza en países en vía de desarrollo: el papel del hotelero y del investigador. Estudios y Perspectivas en Turismo, 17(1), 17-28. Recuperado de http://www.redalyc.org/articulo. oa? id=180713894001

Font, X., Walmsley, A., Cogotti, S., McCombes, L., \& Häusler, N. (2012). Corporate social responsibility: the disclosure-performance gap. Tourism Management, 33(6), 1544-1553. DOI: 10.1016/j. tourman.2012.02.012

Fu, H., Ye, B. H., \& Law, R. (2014). You do well and I do well? The behavioral consequences of corporate social responsibility. International Journal of Hospitality Management, 40, 62-70. DOI: 10.1016/j.ijhm.2014.03.004

Gao, Y. \& Mattila,A. (2014). Improving consumer satisfaction in green hotels: The roles of perceivedwarmth, perceived competence and CSR motive. International Journal of Hospitality Management, 42, 20-31. DOI: 10.1016/j.ijhm.2014.06.003

Garay, L., \& Font, X. (2012). Doing good to do well? Corporate social responsibility reasons, practices and impacts in small and medium accommodation enterprises. International Journal of Hospitality Management, 31(2), 329-337. DOI: 10.1016/j.ijhm.2011.04.013
Garay, L., \& Font, X. (2013). Motivaciones, prácticas y resultados del comportamiento responsable en las pequeñas y medianas empresas turísticas. Revista de Responsabilidad Social de la Empresa, 13(5-1), 51-84.

García F. J. y Del Mar, Y. M. (2007). Aproximación a la incidencia de la responsabilidad socialmedioambiental en el rendimiento económico de la empresa hotelera española. Revista Europea de Dirección y Economía de la Empresa, 16(1), 47-66.

Gardfield, E. (1987). Reviewing review literature. Part 1: definitions and uses ofreviews. Current Contents. Essays of an Information Scientist, 10(18), 113-116.

Glass, R. L., Ramesh, V., \& Vessey, I. (2004). An analysis of research in computing disciplines. Communications of the ACM, 47(6), 89-94. DOI: 10.1145/990680.990686

Glattfelder J. B., \& Battiston, S. (2009). Backbone of complex networks of corporations: The flow of control. Physical Review E, 80(3), 1-24. DOI: 10.1103/PhysRevE.80.036104

González Jiménez de la Espada, G. (2013). Procesos de integración en el sector hotelero español. Revista de Responsabilidad Social de la Empresa 13(5-1), 201-239.

Guédez, V. (2010). Responsabilidad social empresarial. visiones complementarias hacia un modelaje social. Caracas: Cámara VenezolanoAmericana de Comercio e Industria - Venamcham.

Henderson, J. (2007). Corporate social responsibility and tourism: Hotel companies in Phuket, Thailand, after the Indian Ocean tsunami. Hospitality Management, 26(1), 228-239. DOI: 10.1016/j. ijhm.2006.02.001

Holcomb, J. L., Upchurch, R. S., \& Okumus, F. (2007). Corporate Social Responsibility: What are the top hotel companies reporting? 
International Journal of Contemporary Hospitality Management, 19(6), 461-475. DOI: 10.1108/09596110710775129

Huimin, G., \& Ryan, C. (2011). Ethics and corporate social responsibility - An analysis of the views of chinese hotel managers. International Journal of Hospitality Management, 30(4), 875885. DOI: 10.1016/j.ijhm.2011.01.008

Huimin, G., Ryan, C., Li, B., \& Gao, W. (2013). Political connections, guanxi and adoption of CSR policies in the chinese hotel industry: Is there a link? Tourism Management, 34, 231-235. DOI: 10.1016/j.tourman.2012.01.017

Kasim,A. (2004). BESR in the hotel sector: A look at tourists' propensity towards environmentally and socially friendly hotel attributes in Pulau Pinang, Malaysia. International Journal of Hospitality and Tourism Administration, 5(2), 61-83. DOI: 10.1300/J149v05n02_01

Keim, G. D. (1978). Corporate Social Responsibility: An Assessment of the Enlightened SelfInterest Model. Academy of Management Review, 3(1), 32-39. DOI: 10.5465/AMR.1978.4296346

Kitchenham, B. (2004). Procedures for performing systematic reviews. Inglaterra: Keele University.

Khunon, S., \& Muangasame, K. (2013). The differences between local and international chain hotels in CSR management: Empirical findings from a case study in Thailand. Asian Social Science, 9(5), 209-225. DOI: 10.5539/ass.v9n5p209

Kucukusta, D., Mak, A., \& Chan, X. (2013). Corporate social responsibility practices in four and five-star hotels: Perspectives from Hong Kong visitors. International Journal of Hospitality Management, 34, 19-30. DOI: 10.1016/j. ijhm.2013.01.010

Lee, M. D. P. (2008). Review of the theories of corporate social responsibility: It's evolutionary path and the road ahead. International Jour- nal of Management Review, 10(1) 53-73. DOI: 10.1111/j.1468-2370.2007.00226.x

Levy, S. E., \& Park, S. Y. (2011). An Analysis of CSR Activities in the Lodging Industry. Journal of Hospitality and Tourism Management, 18(1), 147-154. DOI: $10.1375 / \mathrm{jhtm} .18 .1 .147$

Lockett, A., Moon, J., \& Visser, W. (2006). Corporate social responsibility in management research: focus, nature, salience and sources of influence. Journal of Management Studies, 43(1), 115-136. DOI: $10.1111 / \mathrm{j} .1467-6486.2006 .00585 . x$

Mackenzie, M., \& Peters, M. (2012). Hospitality managers' perception of corporate social responsibility: An explorative study. Asia Pacific Journal of Tourism Research, 19(1), 1-16. DOI: 10.1080/10941665.2012.742915

Mahon J. F., \& Griffin J. J. (1999). Painting a portrait: A replay. Business and Society, 38(1), 126-133. DOI: $10.1177 / 000765039903800106$

Martos, M. (2011). La responsabilidad social corporativa en la gestión hotelera. Turismo y Sociedad, XXII, 169-184. Recuperado de http:// goo.gl/sFYwJ5

Martínez, P. y Rodríguez del Bosque, I. (2013). CSR and customer loyalty: The roles of trust, customer identification with the company and satisfaction. International Journal of Hospitality Management, 35, 89-99. DOI: 10.1016/j. ijhm.2013.05.009

Martínez, P., Pérez, A. y Rodríguez del Bosque, I. (2013). Responsabilidad social corporativa: definición y práctica en el sector hotelero. El caso de Meliá Hotels. Revista de Responsabilidad Social de la Empresa, 13(5-1), 141-173. Recuperado de http://goo.gl/EMZ0aB

Mattera, M. y Moreno, A. (2012). Strategic implications of corporate social responsibility in hotel industry: A comparative research between NH Hotels and Meliá Hotels International. Higher 
Learning Research Communications, 2(4), 37-53. Recuperado de http://goo.gl/zcYlVv

McGehee, N. G., Wattanakamolchai, S., Perdue, R. R., \& Calvert, E. O. (2009). Corporate social responsibility within the U. S. lodging industry: An exploratory study. Journal of Hospitality \& Tourism Research, 33(3), 417-437. DOI: $10.1177 / 1096348009338532$

Melo, T., Cox Moura-Leite, R., \& Carlton Padgett, R. (2012). Conceptualization of corporate social responsibility by the luxury hotels in Natal/ RN, Brazil. Caderno Virtual de Turismo, 12(2), 152-166. Recuperado de http://www.redalyc.org/ articulo.oa? $\mathrm{id}=115423641004$

Nord, T. (2006). Corporate social responsibility in the hotel industry. A cross culture perspective. Stockholm: Center for Transdisciplinary Environmental Research - CTM, Stockholm University. Recuperado de http://goo.gl/wJOPNU

Peña, D. y Serra, A. (2012). Responsabilidad social empresarial en el sector turístico. Estudio de caso en empresa de alojamiento de la ciudad de Santa Marta, Colombia. Estudios y Perspectivas en Turismo, 21(6), 1456-1480. Recuperado de http:// www.redalyc.org/articulo.oa?id=180725030006

Perdomo, J. y Escobar, A. (2011). La investigación en RSE. Una revisión desde el management. Cuadernos de Administración, 24(43), 193-219. Recuperado de http://www.redalyc.org/articulo. oa? id=20521435009

Pérez, A. (2011). Estudio de la imagen de responsabilidad social corporativa: formación e integración en el comportamiento del usuario de servicios financieros (Tesis doctoral), Universidad de Cantabria. Recuperada de http://goo.gl/ S1wRGA

Rey-Maquieira, J., Aguiló, E., González, A., Ramos, V. y Razumoba, M. (2011). Responsabilidad social empresarial como una herramienta estratégica en las empresas de alojamiento. Palma de
Mallorca: Centro de Investigación y Desarrollo para el Turismo - CITUR e ImageTourism Consultancy and Research.

Robledo, M. (2004). D3D: un enfoque integral de la dirección de empresas. Madrid: Ediciones Días de Santos.

Rodríguez-Antón, J., Alonso-Almeida, M. y Celemín-Pedroche, M. (2013). Responsabilidad social corporativa en las cadenas hoteleras españolas. Un estudio de casos. Revista de Responsabilidad Social de la Empresa, 13(5-1), 15-50. Recuperado de http://goo.gl/UchMn5

Sánchez-Fernández, M. D., Vargas Sánchez, A. y Remoaldo, P. (2014). Institutional context and hotel social responsibility. Kybernetes, 43(3-4), 413-426. DOI: 10.1108/K-12-2013-0267

Sandve, A., \& Øgaard, T. (2014). Exploring the interaction between perceived ethical. Tourism Management 42, 177-180. DOI: 10.1016/j.tourman.2013.11.013

Scanlon, N.L. (2007). An analysis and assessment of environmental operating practices in hotel and resort properties. International Journal of Hospitality Management 26(3), 711-723. DOI: 10.1016/j. ijhm.2006.07.003

Sepúlveda, J. D., Ordóñez, F. y Prada, C. (2014). Perfil de responsabilidad social empresarial del sector hotelero de la ciudad de Bucaramanga, Colombia. Estudios y Perspectivas en Turismo, 23(1), 23-39. Recuperado de http://www.redalyc. org/articulo.oa? id=180729920002

Shaw, B., \& Post, F. R. (1993). A moral basis for corporate philanthropy. Journal of Business Ethics, 12(10), 745-751. Recuperado de http:// goo.g1/3AC $1 \mathrm{X} 3$

Squires, B. P. (1989). Biomedical review articles: What editors want from authors and peer reviewers. Canadian Medical Association Journal, 141(3), 195-197. 
Steiner, G.A. (1972). Social policies for business. California Management Review, 15(2), 17-24.

Tepelus, C. M.(2008). Destination Unknown? The emergence of corporate social responsibility for sustainable development of tourism (Tesis doctoral). Lund University, Lund, Sweden.

Transnational Institute - TNI. (2014). State of Power. Recuperado de http://www.tni.org/stateofpower2014

Trías, M. y García, M. A. (2014). Corporate social responsibility: A study of employee communication in the balearic premium hotels. Athens Journal of Tourism, 1(1), 21-33. Recuperado de http://www.atiner.gr/journals/tourism/2014-1-12-Vilar.pdf

Tsai W., Hsu, L., Chen, C., Lin, W., \& Chen, S. (2010). An integrated approach for selecting corporate social responsibility programs and costs evaluation in the international tourist hotel. International Journal of Hospitality Management, 29(3), 385-396. DOI: 10.1016/j.ijhm.2009.12.001

Tsai, W., Tsang, N., \& Chen, S. (2012). Hotel employees' perceptions on corporate social responsibility: The case of Hong Kong. International
Journal of Hospitality Management, 31(4), 11431154. DOI: 10.1016/j.ijhm.2012.02.002

United Nations Conference on Trade and Development (UNCTAD). (2013). Informe sobre las inversiones en el mundo. Panorama general. Las cadenas de valor mundiales: inversión y comercio para el desarrollo. Nueva York y Ginebra: UNCTAD.

Vaca, R. (2012). Responsabilidad social corporativa en las cadenas hoteleras españolas: factores determinantes de su nivel de desarrollo y consecuencias (Tesis doctoral). Universitat de Huelva, Huelva.

Vitali, S., Glattfelder, J. B., \& Battiston, S. (2011). The Network of Global Corporate Control. PLoS ONE 6(10): e25995. DOI: 10.1371/journal.pone.0025995

Walsh, J. P., Weber, K., \& Margolis, J. D. (2003). Social issues and management: Our lost cause found. Journal of Management, 29(6), 859-881. Recuperado de http://goo.gl/RpypaB

World Travel \& Tourism Council. (2014). Travel \& Tourism. Economic Impact 2014. United States: WTTC. Recuperado de http://goo.gl/svaQFm 